Buches Zeugnis gibt. Stoff und Einteilung ist daher im allgemeinen unverändert geblieben, nur jene Abschnitte haben eine Ausgestaltung erfabren, die in das engere Arbeitsgebjet von 0 . Föppl fallen. So vor allem die über Festigkeitsversuche, über das Verhalten der Materialien bei Schwingungen, über die Kerbwirkung, über die Dämpfungsfähigkeit der Baustoffe u. dgl. AuBerdem wurde das elastische Verhalten von stark gekrümmten Stäben eingebender als in den früheren Auflagen behandelt. Das sind Erweiterungen, die dem Zweck des Buches entsprechen und mit denen man sich einverstanden erklären kann. $K$. Wolf.

H. Driesch, Relativitätstheorie und Philosophie. (Wissen und Wirken, Bd. XIV.) Bei Braun, Karlsruhe 1924. Preis RM 1-.

Die Broschüre enthält eine in knapper, aber klarer Form und in rubigem Tone gehaltene Auseinandersetzung zwischen Philosophie und Physik, die den Verfasser dazu führt, die Relativitätstheorie und insbesondere jhre letzten Konsequenzen als philosophisch unzulässig abzulebnen. Die Einwände decken sich im wesentlichen mit jenen, die schon von $0 . K_{r a u s}$ und anderen erhoben worden sind und mit denen sich Ref. (vgl. diese Ztschr., 1923, Literaturberichte S.53-54) ausführlich auseinandergesetzt hat. Sie sind nach Ansicht des Ref. als längst widerlegt zu betrachten. Es ist interessant, daß gerade jene Ideen, die den meisten Mathematikern und Physikern vom philosophischen Standpunkt aus als besonders befriedigend erscheinen, von seiten vieler Fachphilosophen mit größter Entschiedenheit abgelehnt werden.

Thirring.

J. H. Tummers, Relativitätstheorie nnd Logik. Venlo (Holland) 1924.

Der Verfasser bemerkt richtig, daßB die Relativität der Gleichzeitigkeit eine Folge ist der beiden an den Beginn der Einste in schen Überlegungen gestellten Prinzipien: Relativ.-Prinzip und Prinzip der Konstanz der Lichtgeschwindigkeit. Von diesem Pankt angefangen ist das ganze Heft nur eine gedrängte Darstellung längst widerlegter Vißverständnisse and Fehlschlüsse, deren nähere Besprechung obne Interesse ist.

Halpern.

\title{
D. Gawronsky, Trägheitsgesetz und Aufbau der Relativitätstheorie und Der physikalische Gehalt der speziellen Relativitätstheorie.
}

Es ist nicht leicht, über diese Schriften Näheres zu referieren: Sie enthalten viel Worte, selten Begriffe, nie Tatsachen. Nach der Ansicht des Verfassers sind alle Formeln der Relativitätstheorie richtig, nur ihre physikalische Interpretation ist falsch. Wir zweifeln jedoch an dem Bedürfnis, die physikalischen Neninterpretationen eines Autors näher kennenzulernen, der folgende Sätze sehreibt: "Es ist grundsätzlich falsch, zu sagen, es sei ganz gleich, ob wir das Kilogrammgewieht oder die Erde als bewegt annehmen; diese Vorstellung ist geradezu eine magische, denn durch ein Wort lassen wir ungebeure Energiequanten entstehen und verschwinden. Anders ausgedrückt: Diese Vorstellung ist eine ganz widersinnige." Das heißt: Den Autor stört die Tatsache der Newtonschen Mechanik, daB die kinetische Energie eines Systems von Massenpunkten bei dem Übergang von einem zu einem zweiten Galileischen Koordinatensystem ihren Zahlenwert nicht beibebält.

Halpern.

Die Kultur der Gegenwart - ihre Entwicklung und ihre Ziele. (Herausgegeben von Paul Hinneberg.) Band "Physik" (erster Band der dritten Abteilung des dritten Teils). Zweite, neubearbeitete und erweiterte Auflage nuter Redaktion von Ernst Lecher $\dagger$, Verlag ron B. G. Tenbner, Leipzig und Berlin; 819 Seiten mit 116 Textabbildungen, 1925 .

Innerhalb des groß angelegten Sammelwerkes „Die Kultur der Gegenwart" ist im Jahre 1914. in erster Auflage der Physik-Band erschienen, der in 36 Artikeln eine vorzügliche Übersicht über das gesamte Gebiet der modernen Physik brachte. Unter den 32 Verfassern befanden sich viele Physiker von Weltruhm, wie - um nur einige Namen zu nennen - Planck, H. A. Lorentz, Einstein, Zeeman, Wilhelm Wien. Nicht nur in den Kreisen der Physiker selbst, sondern auch in weiten Laienkreisen hat der Physik-Band der "Kultur der Ge- 